\title{
The multiplicity status of three exoplanet host stars ${ }^{\star}$ (Research Note)
}

\author{
C. Ginski, M. Mugrauer, M. Seeliger, and T. Löhne
}

\author{
Astrophysikalisches Institut und Universitäts-Sternwarte Jena (AIU), University of Jena, Schillergässchen 2, 07745 Jena, Germany \\ e-mail: ginski@astro.uni-jena.de
}

Received 13 July 2013 / Accepted 14 October 2013

\begin{abstract}
Aims. The goal of our ongoing study of exoplanet host stars is to determine the true multiplicity rate of such stars. For this purpose we aim to research a statistically significant and homogeneously analyzed sample of stars around which planets have been found by the radial velocity or the transiting method. Ultimately, we want to determine if there are significant differences between planets in single- and multiple-star systems. This could indicate different formation scenarios. In this research note, we specifically investigate the companionship of three low-mass stellar candidate companions to exoplanet hosts via second epoch imaging and astrometry.

Methods. We used various high-resolution imaging instruments in order to search for low-mass stellar companions to exoplanet host stars. Images were taken at a minimum of two observation epochs to check whether detected companion candidates are co-moving with the primary stars. We used the known photometric data of the primary stars together with theoretical evolutionary models in order to calculate detection limits for all targets and to estimate the mass of confirmed companions.

Results. With our astrometric data we can confirm for the first time that the exoplanet host star HAT-P-8 has a low-mass stellar companion with an absolute magnitude in the $K$-band of $7.37 \pm 0.15 \mathrm{mag}$ and an estimated mass of $0.25 M_{\odot}$. However, in our high-resolution VLT/NACO observation the source appears elongated and could therefore be a close binary. If both of the binary components are equally massive, this binary could have a higher total mass of $\sim 0.35 M_{\odot}$. In addition, we were able to calculate detection limits for the HAT-P-30 system and the HD 44219 system and showed that companion candidates around these objects are most likely background objects.
\end{abstract}

Key words. techniques: high angular resolution - astrometry - planets and satellites: individual: HAT-P-8 - binaries: general

\section{Introduction}

Objects with planetary masses $\leq 13 M_{\text {Jup }}$ have been discovered and confirmed around more than 600 stars with the radial velocity and the transit detection method (see exoplanet.eu by Schneider et al. 2011). However, many of these systems present extreme cases of massive planetary objects at very close separations to their primary stars. Explaining the configurations of these systems is a continued challenge in the development of formation theories. One important parameter that is unknown in most cases is the multiplicity status of the primary star. Given that a large fraction $(46 \% \pm 2 \%$; see, e.g., Raghavan et al. 2010) of solar-type stars in our galaxy is multiple, a significant deviation from this ratio for planet-bearing stars might indicate differences in formation scenarios. Several theoretical studies have been undertaken to explore this relation, but are not yet conclusive. Boss (1998) reported on a numerical study which showed that the presence of a stellar companion can induce a rapid instability even for those planet-bearing discs which are stable in isolation. Nelson et al. (2000) suggested an opposite effect, claiming that planets are unlikely to form in certain binary systems. As was shown by Kley et al. (2000, 2001), the mass accretion and orbital migration rate of exoplanets embedded in a disk is strongly enhanced by a close stellar companion.

* Based on observations obtained at Paranal Observatory in ESO programs 089.C-0638(A) and 090.C-0125(A), as well as archive data of ESO programs 088.D-0473(A), 088.C-0843(B), and 184.D-1152(C).
In our ongoing study we use high-resolution imaging techniques to clarify the multiplicity status of nearby planet host stars (within $250 \mathrm{pc}$ ). For targets on the northern hemisphere we employ the lucky imaging instrument Astralux at the $2.2 \mathrm{~m}$ telescope of the Calar Alto Observatory. The capabilities of this instrument were most recently demonstrated in Ginski et al. (2012), where we clarified the multiplicity status of 70 planet host stars. For targets on the southern hemisphere, we used the NACO high-resolution adaptive optics imager on the ESO-VLT, as well as the SofI wide field imager on the ESO-NTT. Results of this survey are published in several refereed papers, most recently in Mugrauer \& Neuhäuser (2009).

Updates for both our northern and southern hemisphere surveys will be published later this year. To draw statistically significant conclusions from our survey we need to observe a total sample of $\sim 250$ planet host stars. At our current rate of observations we expect to arrive at this sample size within the next two years. A preliminary analysis taking different surveys into account was already published by Roell et al. (2012).

Here we present observations of three exoplanet hosts undertaken with a combination of Astralux and VLT/NACO imaging. The star HD 44219 is a solar analog of spectral type G3 (Kharchenko \& Roeser 2009) at $50.4 \pm 1.9$ pc (van Leeuwen 2007) from the sun. Naef et al. (2010) discovered an $M \sin i=$ $0.58_{-0.06}^{+0.04} M_{\text {Jup }}$ planetary mass object orbiting HD 44219 A at $1.19 \pm 0.02 \mathrm{AU}$, using the radial velocity technique. It was particularly interesting that the eccentricity of the orbit solution 
Table 1. Observation epochs.

\begin{tabular}{|c|c|c|c|c|c|c|}
\hline Date & Target & Instrument & Filter & Exposure time $^{1}[\mathrm{~s}]$ & Pixel scale [mas/pix] & PA of $y$-axis $\left[{ }^{\circ}\right]$ \\
\hline 2011-01-14 & HD 44219 & CAHA $2.2 \mathrm{~m} /$ Astralux & $S D S S-i$ & $50000 \times 0.02954$ & $47.365 \pm 0.135$ & $358.07 \pm 0.12$ \\
\hline 2013-01-24 & HD 44219 & VLT/NACO (S13) & $K \mathrm{~s}$ & $11 \times 120 \times 0.5$ & $13.213 \pm 0.040$ & $0.83 \pm 0.10$ \\
\hline $2011-12-18^{2}$ & HAT-P-30 & NTT/SOFI & $K \mathrm{~s}$ & $6 \times 25$ & $288.017 \pm 0.022$ & $-90.00 \pm 0.04$ \\
\hline $2012-01-23^{2}$ & HAT-P-30 & VLT/HAWK-I & NB 2090 & $11 \times 1.7$ & $106.53 \pm 0.12$ & $-0.12 \pm 0.04$ \\
\hline $2013-01-24$ & HAT-P-30 & VLT/NACO (S13) & $K \mathrm{~s}$ & $10 \times 60 \times 1$ & $13.213 \pm 0.040$ & $0.83 \pm 0.10$ \\
\hline $2011-07-27$ & HAT-P-8 & CAHA $2.2 \mathrm{~m} /$ Astralux & $S D S S-i$ & $50000 \times 0.02954$ & $47.160 \pm 0.066$ & $358.37 \pm 0.23$ \\
\hline $2012-07-23$ & HAT-P-8 & VLT/NACO (S27) & $K \mathrm{~s}$ & $10 \times 120 \times 0.5$ & $27.11 \pm 0.06$ & $0.56 \pm 0.10$ \\
\hline
\end{tabular}

Notes. ${ }^{(1)}$ We give either number of frames and individual exposure time, or the number of frames multiplied by the number of dither positions. (2) Data from public science archives reduced and analyzed by us.

was relatively high for this kind of object at a moderate distance with $0.61 \pm 0.09$.

The star HAT-P-30 (BD+06 1909) is a solar analog of spectral type G0 (Kharchenko \& Roeser 2009). A transiting planetary mass object was discovered around the star by Johnson et al. (2011). The planetary companion has a mass of $0.711 \pm 0.028 M_{\text {Jup }}$ and is located at $0.0419 \pm 0.0005 \mathrm{AU}$ from HAT-P-30 A. The orbital plane of HAT-P-30 b is interesting because it shows a strong tilt $\left(73.5^{\circ} \pm 9.0^{\circ}\right)$ with respect to the spin axis of the host star.

HAT-P-30 b is interesting because its orbital plane shows a strong tilt $\left(73.5^{\circ} \pm 9.0^{\circ}\right)$ with respect to the spin axis of the host star.

The F-type star (Ehrenreich \& Desert 2011) HAT-P-8 (TYC 2757-1152-1) is located at a distance of $230 \pm 15 \mathrm{pc}$ (Latham et al. 2009) from the sun. A $1.34 \pm 0.05 M_{\text {Jup }}$ (Moutou et al. 2010) planetary mass object was discovered via the transiting method at a distance of $0.0449 \pm 0.0007 \mathrm{AU}$ from the star by Latham et al. (2009).

\section{Observations, data reduction and astrometric calibration}

\subsection{Astralux observations}

We observed HD 44219 and HAT-P-8 as part of our ongoing multiplicity survey in the northern hemisphere with the Calar Alto $2.2 \mathrm{~m}$ telescope and the Astralux instrument (Hormuth et al. 2008) in January and July 2011, respectively. To obtain deep images with a high spatial resolution we used the lucky imaging technique (see, e.g., Law et al. 2006). In both cases we took 50000 short exposures with an exposure time of $29.54 \mathrm{~ms}$, each in the SDSS $i$ ' filter (Gunn et al. 1998). To achieve a high signal-to-noise ratio, the gain settings of the instrument were adjusted individually for both targets. On both nights, sky flats were taken in the SDSS $i$ ' filter, as well as 100 bias frames with identical gain settings that were used for the science targets.

For the reduction of the data we measured the Strehl ratio in each image and only chose the top $10 \%$ (i.e., those images with the highest Strehl ratio). Each frame was then flat-fielded and the bias was subtracted before all frames were shifted and co-added.

Since we wanted to obtain precise relative astrometric measurements between primary star and companion candidate, we observed several astrometric calibrators in each night. This included binary stars for which precise HIPPARCOS measurements are available, as well as the center of the globular cluster M 15, for which precise Hubble Space Telescope astrometry is available. The details of the astrometric calibration for both nights are also published in Ginski et al. (2012). The astrometric solution is given in Table 1.

\subsection{NACO observations}

We observed HD 44219 and HAT-P-30 as part of our ongoing multiplicity survey in the southern hemisphere with the ESO-VLT and the NACO instrument (Rousset et al. 2003) in January 2013. In addition we observed HAT-P-8 as a backup target with VLT/NACO in July 2012. All observations were done in the $K_{\mathrm{S}}$-band. We took short exposures of $0.5 \mathrm{~s}$ or $1 \mathrm{~s}$ in order to not overly saturate the bright primary stars. The jitter imaging technique was used to subtract the bright infrared sky background. The stars HAT-P-30 and HD 44219 were observed with the NACO S13 objective for high spatial resolution, while HAT-P-8 could only be observed with the S27 objective owing to program restrictions. All observation parameters are summarized in Table 1.

We took sky flats at the beginning of every night. For the data reduction we used ESO-Eclipse (Devillard 1997). All images were background subtracted, shifted, and co-added.

For astrometric calibration we took images of the globular cluster 47 Tuc, which we compared to the astrometric references taken by the Hubble Space Telescope, as is the standard in our VLT/NACO observations. The astrometric solutions are listed in Table 1. All VLT/NACO images of the primary stars and detected companion candidates are shown in Fig. 1.

\subsection{Archival data}

In addition to our own observations, there was archival data available for HAT-P-30. The star was observed with NTT/SOFI (Moorwood et al. 1998) on 18 December 2011 (program 088.D0473(A)) and with VLT/HAWK-I (Kissler-Patig et al. 2008) on 23 January 2012 (program 088.C-0843(B)). The SOFI images were taken in fast photometry automatic jitter mode using the large field objective in the $K_{\mathrm{s}}$-band. For astrometric calibration we used SOFI data from 20 December 2011 taken in 184.D-1152(C) using the Large field objective in $K$ s-band. The HAWK-I images were only aquisition images taken in the narrow band filter NB2090.

All images were astrometrically calibrated using reference stars from the 2MASS point source catalogue (Cutri et al. 2003). Details of the observations as well as the astrometric solutions are listed in Table 1.

\section{Astrometric analysis}

All astrometric measurements were executed using ESOMIDAS. In order to obtain image coordinates, a Gaussian was fitted to star and companion candidate positions. Image coordinates were then used to calculate separation and position angle 


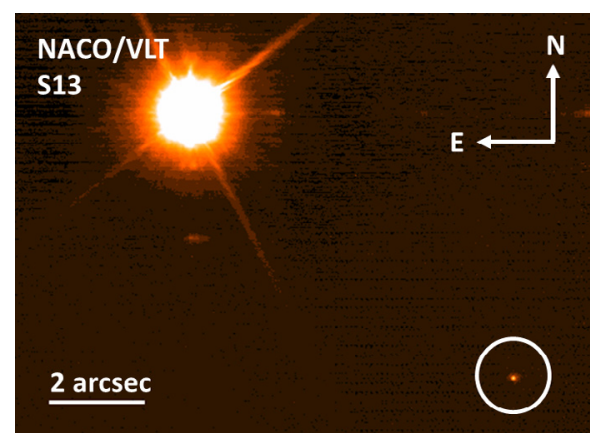

(a) HD 44219 on 2013-01-24

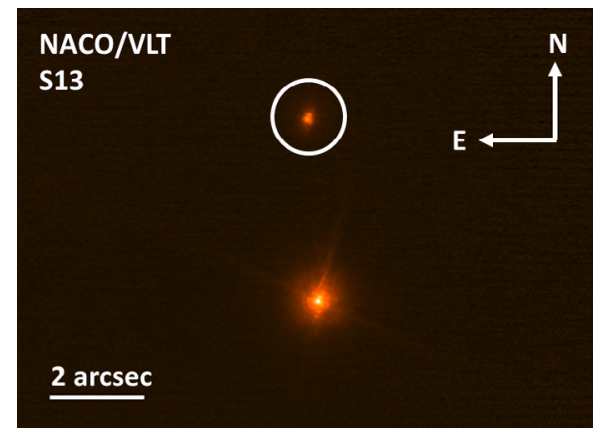

(b) HAT-P-30 on 2013-01-24

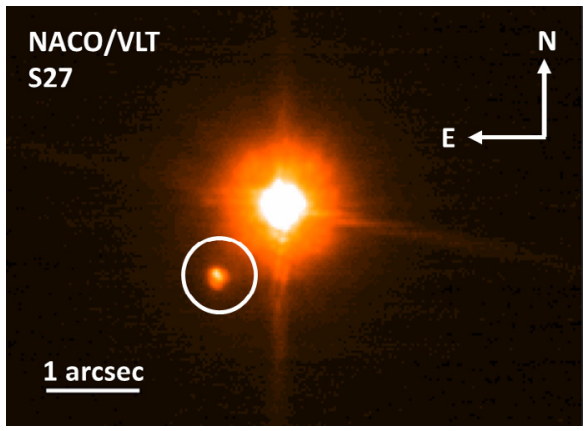

(c) HAT-P-8 on 2012-07-23

Fig. 1. VLT/NACO images of our target stars, all taken in the $K$ s filter. The companion candidates are marked with white circles.

Table 2. Astrometric measurements.

\begin{tabular}{lccc}
\hline \hline Target & Epoch $[\mathrm{yr}]$ & Sep $[\operatorname{arcsec}]$ & $\mathrm{PA}\left[{ }^{\circ}\right]$ \\
\hline HD 44219 & 2011.036 & $8.728 \pm 0.029$ & $230.68 \pm 0.19$ \\
HD 44219 & 2013.063 & $8.766 \pm 0.027$ & $231.68 \pm 0.11$ \\
\hline HAT-P-30 & 2011.964 & $3.833 \pm 0.009$ & $3.81 \pm 0.12$ \\
HAT-P-30 & 2012.063 & $3.842 \pm 0.019$ & $4.19 \pm 0.19$ \\
HAT-P-30 & 2013.063 & $3.815 \pm 0.012$ & $4.15 \pm 0.11$ \\
\hline HAT-P-8 & 2009.828 & $1.027 \pm 0.011$ & $137.3 \pm 0.4$ \\
HAT-P-8 & 2011.571 & $1.043 \pm 0.017$ & $138.84 \pm 0.93$ \\
HAT-P-8 & 2012.560 & $1.039 \pm 0.004$ & $138.49 \pm 0.17$ \\
\hline
\end{tabular}

Notes. ${ }^{(1)}$ As given by Bergfors et al. (2013).

of the companion candidates relative to the primary stars in the image coordinate system. The astrometric solutions for each image were then used to calculate the separation and position angle on the sky plane. The results are listed in Table 2. The uncertainties listed include the uncertainties from the Gaussian fitting as well as the uncertainties of the astrometric solutions.

In Fig. 2 we show the astrometric data points of our targets versus time. The gray oscillating areas show where a nonmoving background object would be located in different observation epochs due to the proper motion of the primary star. The oscillation is introduced by a parallactic effect due to the Earth's revolution around the sun. The dashed lines enclose the area for co-moving objects taking an estimation for orbital motion into consideration. In the case of the position angle, we assumed a face-on circular orbit with a semi-major axis identical to the projected separation of the primary star and companion candidate. In the case of the separation, we assumed an equivalent edge-on orbit.

\section{1. $H D 44219$}

For HD 44219 we compared our initial Astralux observation of January 2011 with a VLT/NACO follow-up observation of January 2013. As can be clearly seen in Fig. 2a, the change in position angle between the two observation epochs is consistent with the background hypothesis and we can exclude the common proper motion hypothesis with $3.7 \sigma$. This is not yet significant in the case of the separation $(0.8 \sigma)$.

\subsection{HAT-P-30}

We observed the HAT-P-30 system in January 2013 with VLT/NACO. In the ESO science archive we found two observations of the system for January 2012 and December 2011, with VLT/HAWK-I and NTT/SOFI, respectively. The companion candidate that we found in our VLT/NACO image was resolved in both archive epochs. In Fig. 2 b we show the astrometric measurements of all three observation epochs. In the case of the position angle, the HAWK-I and SOFI data points show the opposite behavior of the companion candidate. However, the HAWK-I data point might be problematic in position angle, because there were only a few sources visible other than the primary star which could be used for an accurate astrometric calibration. In addition, all images of the sequence were slightly rotated against each other. It is thus likely that we underestimated the uncertainty of the position angle of this data point. The more reliable SOFI data point is more consistent with a non-moving background source than a co-moving companion. Overall we can only reject the common proper motion hypothesis in the case of the position angle with $2.0 \sigma$.

In the case of the separation, both archive epochs are more consistent with the background hypothesis. The common proper motion hypothesis can be rejected with $1.8 \sigma$.

\subsection{HAT-P-8}

For HAT-P-8 we compare our Astralux measurement of July 2011 and our VLT/NACO measurement of July 2012 with the discovery epoch of the companion candidate by Bergfors et al. (2013). The corresponding diagram is shown in Fig. 2c. The uncertainty of our Astralux measurement is significantly higher than the uncertainty of our VLT/NACO measurement because of the small angular separation of the companion candidate and the primary star. In both cases, position angle and separation, we can reject the background hypothesis with a very high significance of $>20 \sigma$ and $>11 \sigma$, respectively.

In addition, we noticed that in our VLT/NACO image the companion candidate appears elongated along a position angle of $\sim 45^{\circ}$, as can be seen in Fig. 3. Comparison with the PSF of the primary star, as well as another faint source at a large angular distance from the primary star, showed that the elongation is only present in the companion candidate. We thus conclude that this is most likely not an adaptive optics effect, but a strong indicator that the companion candidate is actually a binary source.

\section{Detection limits}

We calculated detection limits for all targets with our deep VLT/NACO images. We used the apparent 2MASS $K$-band magnitudes of our targets as well as their distances to compute 

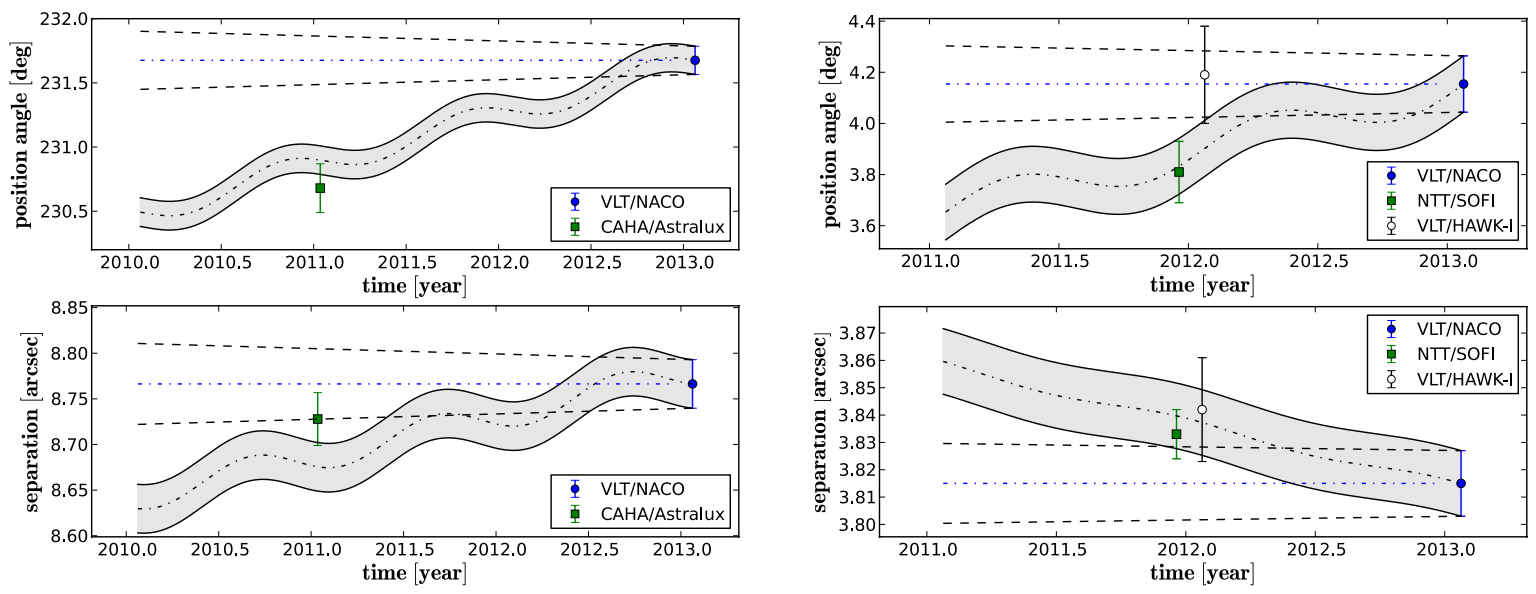

(a) HD 44219

(b) HAT-P-30
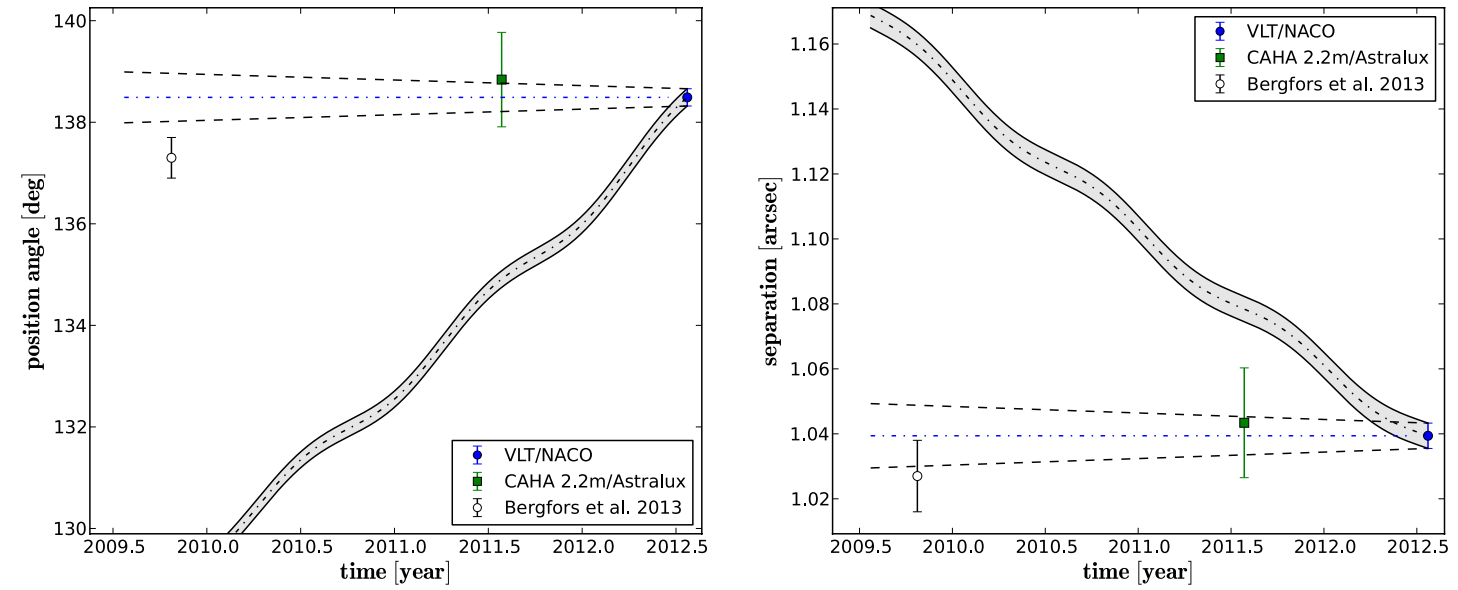

(c) HAT-P-8

Fig. 2. Astrometric measurements of the companion candidates' positions relative to the primary stars versus time. Shown in each case are the separation and the position angle of the companion candidate. The gray area enclosed by the oscillating lines marks the position where a nonmoving background source would be expected due to the proper motion of the primary star on the sky. The oscillation is a parallactic effect introduced by the Earth's revolution around the sun. The area enclosed by the dashed lines marks the position that would be expected for a comoving object, taking orbital motion into account. For the separation, an edge-on circular orbit with a semi-major axis identical to the projected separation of primary and companion candidate was assumed. For the position angle, a respective face-on orbit was considered.

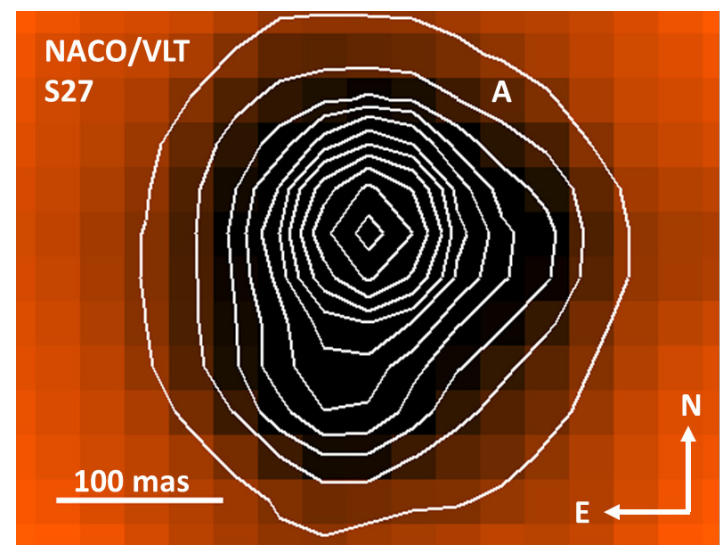

(a) HAT-P-8 A

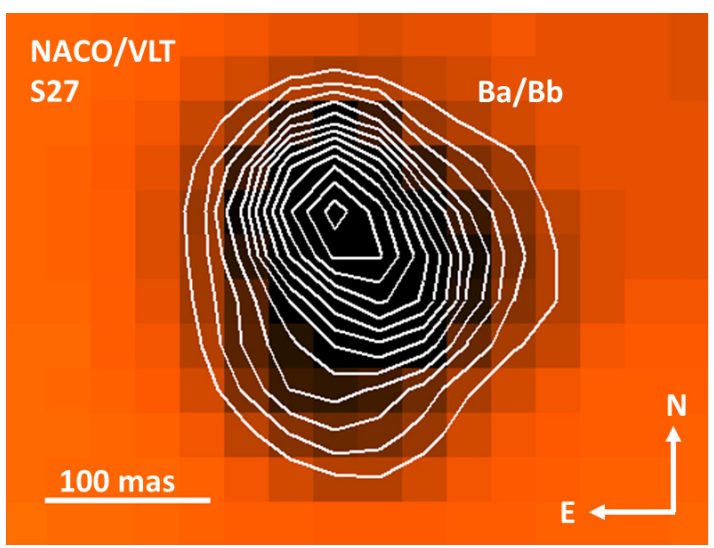

(b) HAT-P-8 B

Fig. 3. Zoomed image of HAT-P-8 A and its co-moving companion. The contour lines clearly show the elongated nature of the B-component along a position angle of $\sim 45^{\circ}$, while the primary star is not elongated in this direction. 
absolute magnitudes in the $K$-band. With the absolute magnitude of the primary and the peak flux measurement, as well as the noise measurement in the images, we are then able to compute limiting absolute magnitudes for our observations. These limiting magnitudes were then compared to the BT-SETTL models by Allard et al. (2011) to translate them into limiting masses, considering the age of the primary star. We generally assume that an object is detectable at a minimum signal-to-noise ratio of 5. Uncertainties in detectable masses include uncertainties of the apparent magnitude, the distance, and the age.

The star HD 44219 shows an apparent magnitude of $6.115 \pm$ $0.017 \mathrm{mag}$ in the $K$-band. Its distance was determined very precisely by HIPPARCos to be $50.4 \pm 1.9$ pc, yielding an absolute magnitude of $K=2.603 \pm 0.084 \mathrm{mag}$. The age of HD 44129 is assumed to be $5.4 \pm 1.5 \mathrm{Gyr}$ as given in Casagrande et al. (2011). Since HD 44219 was slightly saturated in all exposures, we used HAT-P-30 as a flux standard since it was imaged in the same night with the same camera settings. With our VLT/NACO image we can exclude all low-mass stellar companions down to a separation of $0.24 \mathrm{arcsec}$ and up to $5.5 \mathrm{arcsec}$. Since the primary was not put into the center of the field of view in order to image the companion candidate, we can further exclude any lowmass stellar companions with position angles between $152.6^{\circ}$ and $297.2^{\circ}$ up to 10.4 arcsec. Furthermore, we can exclude brown dwarf companions with masses down to $0.053_{-0.003}^{+0.008} M_{\odot}$ in the background limited region outside of 2 arcsec from the primary star, and masses down to $0.069_{-0.004}^{+0.003} M_{\odot}$ as close as 0.5 arcsec.

The star HAT-P-30 exhibits an apparent magnitude of $9.151 \pm 0.023 \mathrm{mag}$ in the $K$-band. Its distance and age are derived by Johnson et al. (2011) from photometry to be $193 \pm 8$ pc and $1 \pm 0.5 \mathrm{Gyr}$, respectively. Given these parameters, we calculate an absolute magnitude of $K=2.723 \pm 0.093 \mathrm{mag}$. With our VLT/NACO images we can exclude all low-mass stellar companions down to a separation of 0.29 arcsec and up to 7.4 arcsec. In addition, we can also in this case exclude brown dwarf companions with masses down to $0.051_{-0.015}^{+0.014} M_{\odot}$ in the background limited area outside of 2 arcsec from the primary star. In 0.5 arcsec from the primary star, we would still have been able to detect brown dwarfs with masses as low as $0.056_{-0.016}^{+0.012} M_{\odot}$.

The star HAT-P-8 has a distance of $230 \pm 15 \mathrm{pc}$ and an age of $3.4 \pm 1 \mathrm{Gyr}$ as derived by Latham et al. (2009) from spectroscopy. It shows an apparent magnitude of $8.953 \pm$ $0.013 \mathrm{mag}$ in the $K$-band. We thus calculate an absolute magnitude of $K=2.34 \pm 0.14 \mathrm{mag}$. In Fig. 4 we show the detection limits of our VLT/NACO observation of HAT-P-8. We can exclude all low-mass stellar companions down to 0.45 arcsec and up to 13.2 arcsec. In the background limited region outside 2 arcsec, we would have been able to detect masses down to $0.065_{-0.006}^{+0.003} M_{\odot}$. We note that we find one additional source in the S27 field of view at a separation of $9.944 \pm 0.022$ arcsec and a position angle of $73.21^{\circ} \pm 0.11^{\circ}$ with respect to the primary star. However, since we could only detect this source in one observation epoch, it is unclear whether it is associated to the HAT-P-8 system or if it is a background object.

\section{Conclusions}

We observed three known exoplanet host stars with VLT/NACO and Astralux at the Calar Alto $2.2 \mathrm{~m}$ Telescope. In our highresolution images we detected three possible low-mass stellar companions to these systems. From our astrometric analysis we conclude that the companion candidate to HAT-P-8, which was

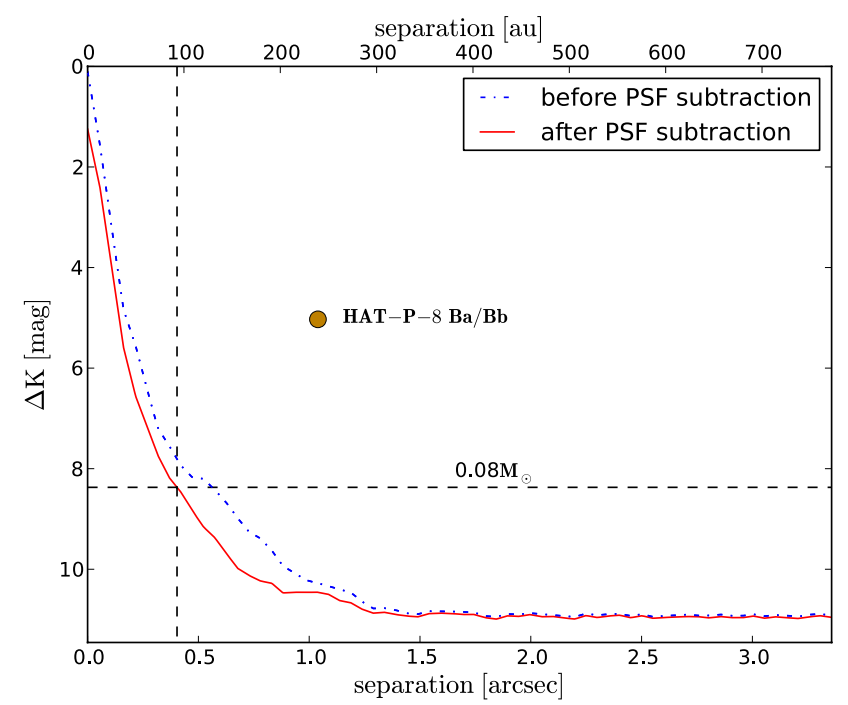

Fig. 4. Dynamic range for the VLT/NACO observation epoch of HAT-P-8. Objects that occupy the parameter space above the solid (red) curve are detectable with a minimum signal-to-noise ratio of 5 . The position of the (possible) binary companion to HAT-P-8 is indicated by the filled circle.

first reported by Bergfors et al. (2013), is co-moving with the primary star and is therefore most likely gravitationally bound. In our VLT/NACO observation epoch we observe an elongation of the companion's PSF that we interpret as strong sign of binarity. Further observations with the NACO S13 objective are planned to confirm this. If we consider all the flux from the companion source we derive an absolute magnitude of $K=7.37 \pm 0.15 \mathrm{mag}$ $(\Delta K=5.03 \pm 0.04 \mathrm{mag})$. By comparison with the BT-SETTL models, we tentatively estimate a mass of $0.25 M_{\odot}$, assuming a single object. If instead we assume two objects of equal mass and thus equal luminosity, we estimate a mass of $\sim 0.175 M_{\odot}$ for each object and therefore a total mass of $\sim 0.35 M_{\odot}$ for the binary.

We also detected one additional source with a separation of $9.944 \pm 0.022 \operatorname{arcsec}$ and a position angle of $73.21^{\circ} \pm 0.11^{\circ}$ with respect to HAT-P-8 A in the NACO S27 field of view, but cannot yet draw a final conclusion as to whether this object is bound or is in the background. However, we evaluated the probability of finding an unrelated object within a 10 arcsec radius of HAT-P-8 by

$P(\Theta, m)=1-\mathrm{e}^{-\pi \Theta^{2} \rho(m)}$,

wherein $\Theta$ is the maximum angular separation and $\rho(m)$ is the surface density of objects down to a limiting magnitude $m$ (as was done by e.g., Correia et al. 2006). To estimate $\rho(m)$ we counted all objects discovered by the WISE survey (Cutri et al. 2012) within a radius of 30 arcmin around HAT-P-8. The result is that, with a probability of $61.8 \%$, there should be at least one unrelated object within 10 arcsec of HAT-P-8. Depending on the spectral type of the hypothetical object, this might only be a lower limit for the probability, because WISE might not be complete down to our limiting magnitude of $\sim 19 \mathrm{mag}$ in the $K$-band. Thus it seems likely that the additional object that we discovered is not related to HAT-P-8, but is in the background.

The companion candidates around HD 44219 and HAT-P-30 that were detected by us are more consistent with background sources after the astrometric analysis. It should be noted, however, that in the case of HAT-P-30 the significance with which we can reject common proper motion with the primary star is 
not yet very high. The star HAT-P-30 should therefore be observed at least one more time in early 2014 to confirm without a doubt the background nature of this source.

We calculated detection limits for all three targets and can exclude additional low-mass stellar companions down to a separation of 0.24 arcsec in the cases of HD 44219 and HAT-P-30, and down to 0.45 arcsec in the case of HAT-P-8.

Acknowledgements. We thank the ESO Paranal Team and ESO Users Support group. We also thank the Calar Alto staff for their help with the observations. This work is partially based on data obtained from the ESO Science Archive Facility. We wish to acknowledge Deutsche Forschungsgemeinschaft (DFG) for grant MU2695 /13-1. This research has made use of the SIMBAD database, operated at CDS, Strasbourg, France. This research has made use of NASA's Astrophysics Data System Bibliographic Services.This publication makes use of data products from the Wide-field Infrared Survey Explorer, which is a joint project of the University of California, Los Angeles, and the Jet Propulsion Laboratory/California Institute of Technology, funded by the National Aeronautics and Space Administration. C.G. expresses special thanks to Donna Keeley for language editing of the paper.

\section{References}

Allard, F., Homeier, D., \& Freytag, B. 2011, ASP Conf. Ser., 448, 91

Baraffe, I., Chabrier, G., Allard, F., \& Hauschildt, P. 2001, ASP Conf. Ser., 243, 571

Bergfors, C., Brandner, W., \& Daemgen, S. 2013, MNRAS, 428, 182

Boss, A. P. 1996, BAAS, 30, 1057

Casagrande, L., Schönrich, R., Asplund, M., et al. 2011, A\&A, 530, A138
Correia, S., Zinnecker, H., Ratzka, T. \& Sterzik, M. F. 2006, A\&A, 459, 909 Cutri, R. M., Skrutskie, M. F., van Dyk, S., et al. 2003, VizieR Online Data Catalog, II/246

Cutri, R. M., et al. 2012, WISE All-Sky Data Release, VizieR Online Data Catalog, II/311

Devillard, N. 1997, The messenger, 87, 19

Ehrenreich, D., \& Desert, J. M. 2011, A\&A, 529, A136

Ginski, C., Mugrauer, M., Seeliger, M., \& Eisenbeiss, T. 2012, MNRAS, 421, 2498

Gunn, J. E., Carr, M., Rockosi, C., et al. 1998, AJ, 116, 3040

Hormuth, F., Hippler, S., Brandner, W., Wagner, K., \& Henning, T. 2008, Proc. SPIE, 7014, 138

Johnson, M., Sollerman, J., Fynbo, J. P. U., et al. 2011, ApJ, 735, 24

Kharchenko, N. V., \& Roeser, S. 2009, VizieR Online Data Catalog, I/280B

Kissler-Patig, M., Pirard, J.-F., Casali, M., Moorwood, A., et al. 2008, A\&A, 491, 941

Kley, W. 2000, IAU Symp., 200, 211

Kley, W. 2001, IAU Symp., 200, 511

Latham, D. W., Bakos, G. Á., Torres, G., et al. 2009, ApJ, 704, 1107

Law, N. M., Mackay, C. D., \& Baldwin, J. E. 2006, A\&A, 446, 739

van Leeuwen, F. 2007, Hipparcos, the New Reduction of the Raw Data, Astrophys. Space Sci. Lib., 350

Naef, D., Mayor, M., Lo Curto, G., et al. 2010, A\&A, 523, A15

Moorwood, A., Cuby, J. G., \& Lidman, C. 1998, The Messenger, 91,9

Moutou, C.,Díaz, R. F., Udry, S., et al. 2011, A\&A, 533, A113

Mugrauer, M., \& Neuhäuser, R. 2009, A\&A, 494, 373

Nelson, A. F. 2000, ApJ, 537, L65

Raghavan, D., McAlister, H. A., Henry, T. J., et al. 2010, ApJ, 190, 1

Roell, T., Neuhäuser, R., Seifahrt, A., \& Mugrauer, M. 2012, A\&A, 542, A92

Rousset, G., Lacombe, F., Puget, P., et al. 2003, SPIE, 4839, 140

Schneider, J., Dedieu, C., Le Sidaner, P., Savalle, R., \& Zolotukhin, I. 2011, A\&A, 532, A79 